\title{
Interstitial-Mediated Diffusion in Germanium under Proton Irradiation
}

\author{
H. Bracht, ${ }^{1 *}$ S. Schneider, ${ }^{1}$ J. N. Klug, ${ }^{2}$ C. Y. Liao, ${ }^{3}$ J. Lundsgaard Hansen, ${ }^{4}$ E. E. Haller, ${ }^{3}$ A. Nylandsted Larsen, ${ }^{4}$ \\ D. Bougeard, ${ }^{5}$ M. Posselt, ${ }^{6}$ and C. Wündisch ${ }^{6}$ \\ ${ }^{1}$ Institute of Materials Physics, University of Münster, Wilhelm-Klemm-Straße 10, D-48149 Münster, Germany \\ ${ }^{2}$ RUBION, Ruhr-Universität Bochum, Universitätsstraße 150, D-44780 Bochum, Germany \\ ${ }^{3}$ MS\&E Department, University of California at Berkeley, and Lawrence Berkeley National Laboratory, \\ 1 Cyclotron Road, Berkeley, California 94720, USA \\ ${ }^{4}$ Department of Physics and Astronomy, Aarhus University, Ny Munkegade, DK-8000 Aarhus, Denmark \\ ${ }^{5}$ Walter Schottky Institute, Technical University of Munich, Am Coulombwall 3, D-85748 Garching, Germany \\ ${ }^{6}$ Forschungszentrum Dresden-Rossendorf, Institute of Ion Beam Physics and Materials Research, D-01314 Dresden, Germany
} (Received 26 August 2009; published 16 December 2009)

\begin{abstract}
We report experiments on the impact of $2.5 \mathrm{MeV}$ proton irradiation on self-diffusion and dopant diffusion in germanium (Ge). Self-diffusion under irradiation reveals an unusual depth independent broadening of the Ge isotope multilayer structure. This behavior and the observed enhanced diffusion of $B$ and retarded diffusion of $P$ demonstrates that an interstitial-mediated diffusion process dominates in $\mathrm{Ge}$ under irradiation. This fundamental finding opens up unique ways to suppress vacancy-mediated diffusion in $\mathrm{Ge}$ and to solve the donor deactivation problem that hinders the fabrication of Ge-based nanoelectronic devices.
\end{abstract}

Over the past few years the elemental semiconductor Ge has been the subject of many experimental [1-13] and theoretical investigations [14-25] to elucidate the electronic and diffusion properties of point defects as well as their interaction. Understanding these properties helps to develop strategies for efficient defect engineering that are crucial for the fabrication of the next generation of nanoelectronic devices. Utilizing $\mathrm{Ge}$ instead of silicon ( $\mathrm{Si}$ ) for complementary metal oxide semiconductors (CMOS) technology one can take advantage of the higher electron and hole mobilities in Ge compared to Si [26]. Whereas the $p$-channel Ge-MOSFET (metal oxide semiconductor fieldeffect transistor) made of heavily $B$ doped source and drain regions was already demonstrated [27], the $n$-channel MOSFET remains a challenge due to the enhanced diffusion of $n$-type dopants such as $P$, As, and Sb under extrinsic doping conditions and the deactivation of the donors for concentrations exceeding $10^{19} \mathrm{~cm}^{-3}[7,9,27]$. The enhanced diffusion is a consequence of the singly negatively charged donor-vacancy $(A V)^{-}$pair that mediates donor diffusion in $\mathrm{Ge}$ according to the reaction [7,9]

$$
(A V)^{-} \leftrightarrow A_{s}^{+}+V^{2-}
$$

where $A_{s}^{+}$and $V^{2-}$ are the singly positively charged substitutional donor with $A \in\{\mathrm{P}, \mathrm{As}, \mathrm{Sb}\}$ and the doubly negatively charged vacancy $\left(V^{2-}\right)$, respectively. The deactivation is related to the formation of inactive donorvacancy clusters whose formation is favored due to Coulomb attraction between $A_{s}^{+}$and $(A V)^{-}$via the reaction [9]

$$
(A V)^{-}+A_{s}^{+} \leftrightarrow\left(A_{2} V\right)^{0} .
$$

The formation of $A_{2} V$ and even bigger clusters $A_{n} V_{m}$ is consistent with the predictions of density functional theory calculations [23]. Reactions (1) and (2) indicate that the donor-vacancy pair mediates both the diffusion and deactivation of $n$-type dopants in Ge. Effective defect engineering that aims to suppress the enhanced diffusion and deactivation of donors in Ge should reduce the concentration of the $A V$ pairs. In this letter we demonstrate that defect engineering with $\mathrm{Ge}$ interstitials makes it possible to effectively suppress the enhanced diffusion of donor atoms.

The formation enthalpy of interstitials is predicted to be much higher than that of vacancies in Ge $[19,20]$. Accordingly, atomic transport in Ge under thermal equilibrium is mainly mediated by vacancies [7]. However, interstitials in Ge can be formed under irradiation as demonstrated in previous studies $[10,13]$. Stimulated by these results and the understanding on the evolution of interstitial clusters in Si [28], the impact of implantation damage on the diffusion in Ge was investigated by several research groups $[11,12,29]$. However, post anneals of dopant implanted Ge did not reveal any significant TED or transient retarded diffusion (TRD) [29,30]. Instead, it is generally observed, that the implantation damage in Ge recovers fast even at low temperatures $[29,30]$.

In order to investigate the impact of interstitials on diffusion in Ge we performed diffusion experiments under proton irradiation. For these experiments we utilized a single crystalline Ge isotope multilayer structure consisting of 20 alternating enriched ${ }^{70} \mathrm{Ge}(96 \%$ enrichment) and natural Ge layers. With a thickness of $15 \mathrm{~nm}$ for each layer a total thickness of $300 \mathrm{~nm}$ is obtained. The isotope struc- 
ture was grown by molecular beam epitaxy (MBE) on a (100)-oriented $\mathrm{Ge}$ wafer at $250{ }^{\circ} \mathrm{C}$. The distribution of ${ }^{74} \mathrm{Ge}$ within the multilayer measured with secondary ion mass spectrometry (SIMS) is illustrated in Fig. 1. In addition, we used a MBE grown structure with six $B$-doped Ge layers. The $B$-doped layers are each about $25 \mathrm{~nm}$ thick and separated by $100 \mathrm{~nm}$ undoped Ge. With a $100 \mathrm{~nm}$ thick undoped Ge cap and a buffer layer of $100 \mathrm{~nm}$ the total thickness of the single crystalline $B$-doped Ge structure is about $800 \mathrm{~nm}$. A SIMS analysis of the asgrown $B$-doped structure is illustrated in Fig. 2. Finally, a Ge sample implanted with $P$ at $30 \mathrm{keV}$ to a dose of $3 \times 10^{15} \mathrm{~cm}^{-2}$ was prepared. In order to prevent outdiffusion of $P$ during annealing a $10 \mathrm{~nm}$ thick $\mathrm{SiO}_{2}$ layer was sputter deposited on the Ge sample before implantation. A SIMS analysis of the as-implanted $P$ profile is illustrated in Fig. 3.

Samples with lateral dimensions of $4 \times 4 \mathrm{~mm}^{2}$ were cut from the as-grown or as-implanted Ge wafers, thinned to a thickness of $30( \pm 5) \mu \mathrm{m}$, and polished with Nalco 2360 (Bucher AG, Switzerland) to obtain a scratch free and specular surface on the back. The samples were mounted on a graphite holder and fixed via a graphite plate with a circular aperture of $3 \mathrm{~mm}$ in diameter. In this way the outer

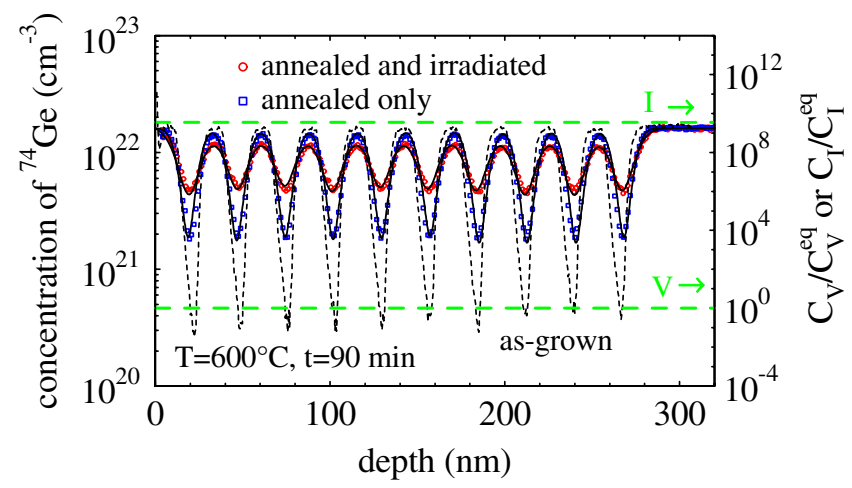

FIG. 1 (color online). Concentration profiles of ${ }^{74} \mathrm{Ge}$ measured with TOF-SIMS before (as-grown: thin dashed line) and after annealing (symbols) at the temperature and time indicated. Only every 6th data point is shown for clarity. The ${ }^{74} \mathrm{Ge}$ profile from the covered part of the sample (blue squares) represents Ge diffusion under equilibrium condition. The corresponding black solid line shows the expected Ge diffusion profile for selfdiffusion under equilibrium conditions at $600{ }^{\circ} \mathrm{C}$ taking into account published self-diffusion data [34]. The ${ }^{74} \mathrm{Ge}$ profile obtained from the proton irradiated area of the sample (red circles) indicates an enhanced Ge diffusion under irradiation with respect to equilibrium conditions. The corresponding black solid line shows the numerical simulation of self-diffusion under irradiation assuming different boundary conditions for vacancies and self-interstitials (see text for details). The corresponding concentration profiles of vacancies and self-interstitials normalized to the respective equilibrium concentration (see right $y$ axis) are displayed by the lower and upper green dashed lines, respectively. part of the Ge sample was covered with graphite and not exposed to the proton beam. The graphite holder was placed on a boron nitride heating plate that enables heating of the Ge sample during irradiation. The temperature was controlled with a thermocouple mounted $1 \mathrm{~mm}$ below the sample in the graphite holder. The whole sample holder was attached to a high vacuum chamber. Protons of 2.5 MeV were supplied via a beam line from a dynamitron accelerator. The beam was defocused and swept to achieve a homogeneously irradiated circular area with a diameter of $1 \mathrm{~cm}$. An electron suppression, which consists of a negatively biased $(600 \mathrm{~V})$ screen, ensures that the measurement of the proton current is not hampered by secondary electrons. Proton irradiations were performed at 570 and $600{ }^{\circ} \mathrm{C}$ with a proton flux of $1.5 \mu \mathrm{A}$. The high energy of the protons assures that the protons penetrate through the Ge sample as this is ascertained by simulations of the "stopping and range of ions in matter" (SRIM [31]). After annealing under proton irradiation the respective concentration profiles of ${ }^{74} \mathrm{Ge}, B$, and $P$ were measured with SIMS. The depth of the SIMS craters was determined using an optical profilometer.

Figure 1 shows the concentration profile of ${ }^{74} \mathrm{Ge}$ after proton irradiation at $600{ }^{\circ} \mathrm{C}$ for $90 \mathrm{~min}$ (see red circles). The broadening of the isotope structure under thermal equilibrium measured with SIMS at the outer part of the samples is illustrated by the blue squares. The Ge profile from the proton exposed inner part of $\mathrm{Ge}$ isotope sample

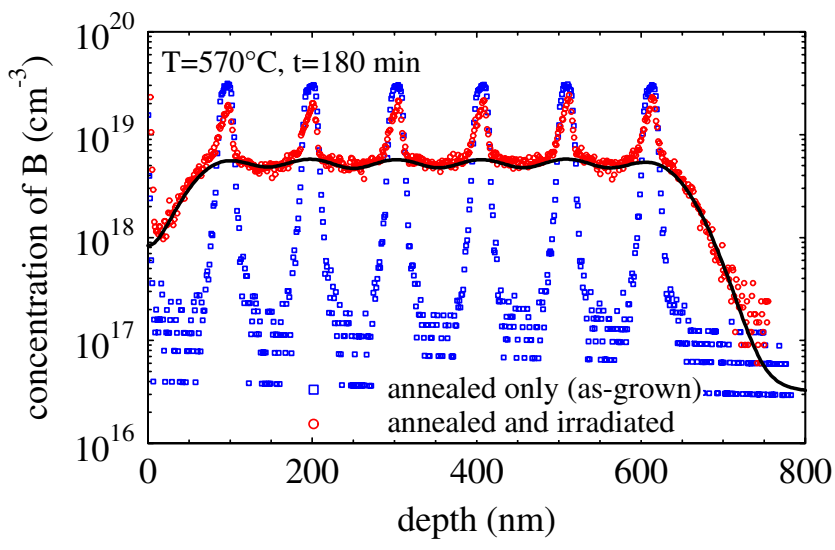

FIG. 2 (color online). Concentration profiles of $B$ in Ge measured with TOF-SIMS after diffusion annealing at the temperature and time indicated. For clarity only every 6th data point is shown. The $B$ profile from the covered part of the sample (blue squares) represents $B$ diffusion under equilibrium conditions. This profile equals the as-grown profile because $B$ diffusion in thermal equilibrium is very slow [4]. The $B$ profile obtained from the proton irradiated area of the sample (red circles) indicates an enhanced $B$ diffusion under irradiation with respect to equilibrium conditions. The black solid line shows a simulation of $B$ diffusion based on Fick's second law with a concentration independent effective diffusion coefficient of $7.5 \times$ $10^{-16} \mathrm{~cm}^{2} \mathrm{~s}^{-1}$ which exceeds the equilibrium diffusion of $B$ by several orders of magnitude. 


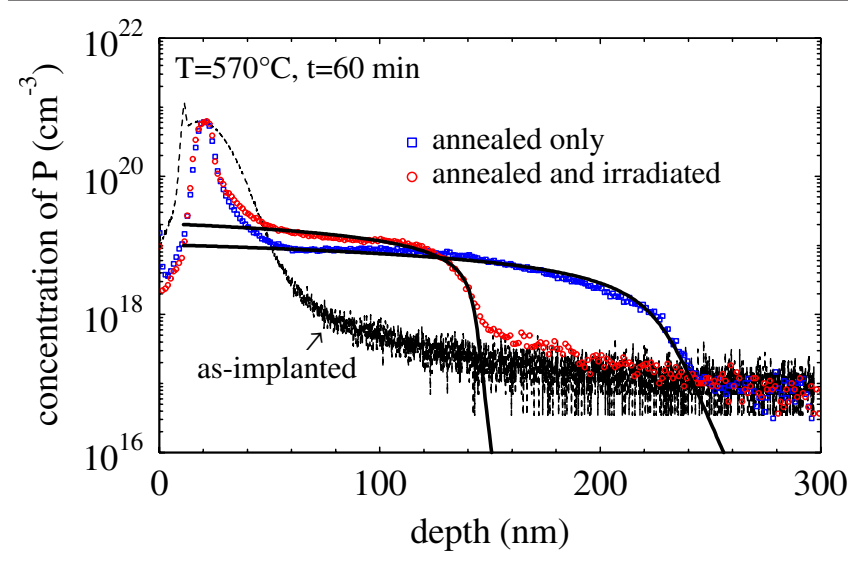

FIG. 3 (color online). Concentration profiles of $P$-implanted Ge measured with TOF-SIMS before (thin black dashed line) and after diffusion annealing (symbols) at the temperature and time indicated. Only every 6th data point is shown for clarity. The $P$ profile from the covered part of the sample (blue squares) represents $P$ diffusion under equilibrium conditions. The corresponding black solid line shows the expected $P$ profile for diffusion under equilibrium conditions at $600{ }^{\circ} \mathrm{C}$ taking into account published data [7]. The $P$ profile obtained from the proton irradiated area of the sample (red circles) indicates a retarded $P$ diffusion under irradiation with respect to equilibrium conditions. The corresponding black solid line is a numerical simulation of the concentration dependent $P$ diffusion that reveals a factor of 20 lower effective diffusion coefficient of $P$ under irradiation compared to equilibrium conditions. Note, the $10 \mathrm{~nm}$ thick $\mathrm{SiO}_{2}$ cap layer was not removed for the SIMS analysis and considered in the simulations of $P$ diffusion by an offset in the profile. The high $P$ concentrations in the peak region of the implanted $P$ profiles likely reflect the formation of $P$ clusters.

shows a more pronounced intermixing. This unambiguously demonstrates an enhanced self-diffusion under irradiation. The homogenous broadening of the isotope structure under irradiation is, however, very unusual. This reveals that no gradient in the concentration of the native point defects versus depth exists. Usually the free surface acts as sink for vacancies and self-interstitials that are formed by proton irradiation in equal numbers and concentrations (see, e.g., [32]). The homogenous broadening of the Ge isotope structures reflects that thermal equilibrium is disturbed at the surface. The Ge profile alone can not tell us whether this holds for both vacancies and self-interstitials or just for one of these defects. In order to identify the surface condition, we performed additional experiments with $B$-doped and $P$-implanted $\mathrm{Ge}$ samples. Under thermal equilibrium the diffusion of $B$ in Ge is very low; its diffusion coefficient is several orders of magnitude smaller than the self-diffusion coefficient [4]. This behavior is in accord with theoretical calculations [18] that predict a repulsive interaction between substitutional $B$ and the vacancy. Annealing of the $B$-doped multilayer structure at $570{ }^{\circ} \mathrm{C}$ under proton irradiation leads to a very strong intermixing as illustrated by Fig. 2. For com- parison, the $B$ profile beneath the outer part of the $\mathrm{Ge}$ sample, covered by a graphite plate, did not show any broadening at all, i.e., the as-grown $B$ profile is reproduced. The strong enhancement of $B$ diffusion under irradiation is likely caused by interstitials whose concentration under irradiation exceeds their thermal equilibrium concentration by several orders of magnitude. The $B$ spikes with a concentration of $3 \times 10^{19} \mathrm{~cm}^{-3}$ that interfere with the diffusion profile reveal an immobile fraction of $B$ in Ge. This is similar to the behavior of $B$ in $\mathrm{Si}$ where $B$-interstitial clusters have been identified as the origin of the immobile $B$ fraction [28]. The concept of an interstitialmediated diffusion in Ge under irradiation is confirmed by the diffusion behavior of $P$. $P$ diffusion is mediated by donor-vacancy pairs via the vacancy mechanism [see Eq. (1)] [7,9]. In the case interstitials dominate under proton irradiation the diffusion of $P$ should be retarded. Indeed, Fig. 3 demonstrates a reduced penetration depth of $P$ compared to the profile measured beneath the covered part of the same Ge sample.

The results of the impact of proton irradiation on selfand dopant diffusion in Ge show that the Ge surface is not an efficient sink for interstitials. The interstitials created during irradiation are assumed to be reflected at the $\mathrm{Ge}$ surface. The vacancies that are produced in equal number are annihilated at the surface and in the bulk via recombination with interstitials. During irradiation the concentration of interstitials increases and, finally, dominates over the vacancy concentration, and promotes an interstitialmediated diffusion. This diffusion behavior can be modeled on the basis of a continuum theoretical approach that considers the formation of point defects by irradiation and their annihilation via the Frenkel pair reaction [10]. Numerical simulations provide an accurate description of the experimental Ge profile when reflecting boundary conditions for the Ge interstitials are assumed. The calculated profile is given by the black solid line in Fig. 1. Analysis of the $B$ profile reveals an enhancement factor of $2.8 \times 10^{7}$ for $B$ diffusion under irradiation compared to equilibrium diffusion [4] (see Fig. 2). The analysis of the $P$ profiles shown in Fig. 3 yields a retardation factor of about 20 for $P$ diffusion under irradiation.

The inability of the Ge surface to annihilate interstitials leads to the interstitial-mediated diffusion under irradiation. Recently, interstitials in Ge were formed via electron irradiation and directly observed by means of HRTEM [33]. Their direct observation would be hardly possible in the case the Ge surface is a perfect sink for interstitials. The property of the Ge surface seems to hold for bare surfaces like those prepared in Ref. [33] and surfaces covered with impurities. Our samples were annealed and proton irradiated in a vacuum of about $10^{-6}$ mbar. Under these conditions they certainly do not exhibit bare surfaces. This also applies to the $P$-implanted sample with a thin $\mathrm{SiO}_{2}$ layer on top. 
In conclusion, our experiments on self- and dopant diffusion in Ge under proton irradiation clearly demonstrate that interstitial-mediated diffusion is favored under irradiation that promotes $B$ diffusion and retards $P$ diffusion. These findings open up new strategies to effectively suppress vacancy-mediated diffusion in Ge that prevails under thermal equilibrium conditions. Thermal treatments under irradiation are proposed to solve the diffusion and also the doping issues that limit today the fabrication of $\mathrm{Ge}$ $n$-channel MOSFET's.

This work was supported by the Deutsche Forschungsgemeinschaft under contract number BR 1520/6-2.

*Corresponding author.

bracht@uni-muenster.de

[1] Germanium-Based Technologies-From Materials to Devices, edited by C. Claeys and E. Simoen (Elsevier, Amsterdam, 2007).

[2] H. Häßlein, R. Sielemann, and C. Zistl, Phys. Rev. Lett. 80, 2626 (1998).

[3] V.P. Markevich, I.D. Hawkins, A. R. Peaker, K. V. Emtsev, V. V. Emtsev, V. V. Litvinov, F. L. I. Murin, and L. Dobaczewski, Phys. Rev. B 70, 235213 (2004).

[4] S. Uppal, A.F.W. Willoughby, J.M. Bonar, N.E. B. Cowern, T. Grasby, R. J.H. Morris, and M. G. Dowsett, J. Appl. Phys. 96, 1376 (2004).

[5] C.E. Lindberg, J. Lundsgaard Hansen, P. Bomholt, A. Mesli, K. Bonde Nielsen, A. Nylandsted Larsen, and L. Dobaczewski, Appl. Phys. Lett. 87, 172103 (2005).

[6] V. Emtsev, Mater. Sci. Semicond. Process. 9, 580 (2006).

[7] S. Brotzmann and H. Bracht, J. Appl. Phys. 103, 033508 (2008).

[8] A. Mesli, L. Dobaczewski, K. Bonde Nielsen, V. Kolkovsky, M. Christian Petersen, and A. Nylandsted Larsen, Phys. Rev. B 78, 165202 (2008).

[9] S. Brotzmann, H. Bracht, J. Lundsgaard Hansen, A. Nylandsted Larsen, E. Simoen, E. E. Haller, J. S. Christensen, and P. Werner, Phys. Rev. B 77, 235207 (2008).

[10] S. Schneider, H. Bracht, M. C. Petersen, J. Lundsgaard Hansen, and A. Nylandsted Larsen, J. Appl. Phys. 103, 033517 (2008).

[11] G. Impellizzeri, S. Mirabella, E. Bruno, A. M. Piro, and M. G. Grimaldi, J. Appl. Phys. 105, 063533 (2009).

[12] P. Tsouroutas, D. Tsoukalas, I. Zergioti, N. Cherkashin, and A. Claverie, J. Appl. Phys. 105, 094910 (2009).

[13] E. Bruno, S. Mirabella, G. Scapellato, G. Impellizzeri, A. Terrasi, F. Priolo, E. Napolitani, D. De Salvador, M. Mastromatteo, and A. Carnera, Phys. Rev. B 80, 033204 (2009).

[14] A. Fazzio, A. Janotti, Antonio J. R. da Silva, and R. Mota, Phys. Rev. B 61, R2401 (2000).
[15] M. Dionízio Moreira, R. H. Miwa, and P. Venezuela, Phys. Rev. B 70, 115215 (2004).

[16] H. Höhler, N. Atodiresei, K. Schroeder, R. Zeller, and P. H. Dederichs, Phys. Rev. B 71, 035212 (2005).

[17] J. Coutinho, S. Öberg, V.J. B. Torres, M. Barroso, R. Jones, and P.R. Briddon, Phys. Rev. B 73, 235213 (2006).

[18] A. Chroneos, B. P. Uberuaga, and R. W. Grimes, J. Appl. Phys. 102, 083707 (2007).

[19] J. Vanhellemont, P. Śpiewak, and K. Sueoka, J. Appl. Phys. 101, 036103 (2007).

[20] A. Carvalho, R. Jones, C. Janke, J. P. Goss, P. R. Briddon, J. Coutinho, and S. Öberg, Phys. Rev. Lett. 99, 175502 (2007).

[21] A. Chroneos, H. Bracht, R.W. Grimes, and B.P. Uberuaga, Appl. Phys. Lett. 92, 172103 (2008).

[22] J. Coutinho, C. Janke, A. Carvalho, S. Öberg, V. J. B. Torres, R. Jones, and P.R. Briddon, Defect and Diffusion Forum 273-276, 93 (2008).

[23] A. Chroneos, R.W. Grimes, B. P. Uberuaga, and H. Bracht, Phys. Rev. B 77, 235208 (2008).

[24] C. Janke, R. Jones, S. Öberg, and P. R. Briddon, Phys. Rev. B 77, 195210 (2008).

[25] C. Janke, R. Jones, S. Öberg, and P. R. Briddon, Phys. Rev. B 77, 075208 (2008).

[26] S. M. Sze, Physics of Semiconductor Devices (John Wiley and Sons, New York, 2001).

[27] D. P. Brunco, B. De Jaeger, G. Eneman, J. Mitard, G. Hellings, A. Satta, V. Terzieva, L. Souriau, F.E. Leys, G. Pourtois, M. Houssa, G. Winderickx, E. Vrancken, S. Sioncke, K. Opsomer, G. Nicholas, M. Caymax, A. Stesmans, J. Van Steenbergen, P.W. Mertens, M. Meuris, and M. M. Heyns, J. Electrochem. Soc. 155, H552 (2008).

[28] S. C. Jain, W. Schoenmaker, R. Lindsay, P. A. Stolk, S. Decoutere, M. Willander, and H.E. Maes, J. Appl. Phys. 91, 8919 (2002).

[29] A. Satta, E. Simoen, T. Janssens, T. Clarysse, B. De Jaeger, A. Benedetti, I. Hoflijk, B. Brijs, M. Meuris, and W. Vandervorst, J. Electrochem. Soc. 153, G229 (2006).

[30] M. Posselt, B. Schmidt, W. Anwand, R. Grötzschel, V. Heera, A. Mücklich, C. Wündisch, W. Skorupa, H. Hortenbach, S. Gennaro, M. Bersani, D. Giubertoni, A. Möller, and H. Bracht, J. Vac. Sci. Technol. B 26, 430 (2008).

[31] J. F. Ziegler, J. P. Biersack, and U. Littmark, The Stopping and Range of Ions in Solids, Stopping and Ranges of Ions in Matter (Pergamon, New York, 1984), Vol. 1.

[32] H. Bracht, J. Fage Pedersen, N. Zangenberg, A. Nylandsted Larsen, E.E. Haller, G. Lulli, and M. Posselt, Phys. Rev. Lett. 91, 245502 (2003).

[33] D. Alloyeau, B. Freitag, S. Dag, Lin W. Wang, and C. Kisielowski, Phys. Rev. B 80, 014114 (2009).

[34] E. Hüger, U. Tietze, D. Lott, H. Bracht, D. Bougeard, E. E. Haller, and H. Schmidt, Appl. Phys. Lett. 93, 162104 (2008). 